Aus der kaiserlichen chirurgischen Universitätsklinik Kyoto, Japan (Prof. H. I t o).

\title{
Über die lymphatische Leukämie, mit besonderer Berücksichtigung ihrer großzelligen Form ${ }^{1}$ ).
}

\author{
Von Dr. H. Nakamura, Assistent der Klinik.
}

(Mit 3 Abbildungen auf Tafel I.)

Manche Frage bezüglich der Pathogenese der Leukämie harrt immer noch der Lösung. Als ein fundamentaler Unterschied zwischen den leukämischen bzw. pseudoleukämischen Tumoren und den echten malignen Geschwülsten ist seit langem die lokale Begrenzung der ersteren, das schrankenlose Wachstum der letzteren angesehen worden. Aber selbst dieses Merkmal ist nicht mehr in vollem Umfange aufrecht $z u$ halten, seitdem innige, höchst auffallende Beziehungen zwischen den auf einfacher Hyperplasie beruhenden leukämischen Tumoren und den Sarkomen bekannt geworden sind. Noch inniger sind die Beziehungen der letzteren zur Pseudoleukämie. Es wurden ja bereits von Virchow alle mit aleukämischem Blutbefunde einhergehenden, klinisch bösartigen, multiplen Lymphdrüsentumoren als Lymphosarkome bezeichnet. B a n ti sieht die Leukämie als eine systematische lymphadenoide Sarkomatose der lympho- und hämatopoëtischen Organe an. $\mathrm{Much}$ behauptet, daß die lymphatische Leukämie den Infektionskrankheiten zuzurechnen sei. Und Barrenscheen züchtete in einem Fall akuter Leukämie intra vitam aus dem Blute Staphylococcus aureus. Bei diesen Meinungsdifferenzen in bezug auf das Wesen der Leukämie sei uns gestattet, einen kasuistischen Beitrag zu liefern. Der Fall ist kurz folgender:

Anamncse: 4 Jahre altes Mädchen. Zum richtigen Termin glatt geboren. Geistige und körperliche Entwicklung normal. Schun

I) Diese Arbeit wurde noch vor Ausbruch des Europäischen Krieges für die Zeitschrift angenommen.

Die Schriftleitung. 
Masern durchgemacht. Am 20. IV. I9I3 fiel sie um und stieß sich die Stirngegend auf einen harten Boden. Es stellt sich daselbst bald darauf eine bohnengroße, nicht druckempfindliche Anschwellung ein, die sich nachher allmählich vergrößerte und schwarz-rötlich verfärbte. Je eine bohnengroße Anschwellung entstand am I I. nächsten Monats an der linken und io Tage später an der rechten Retromandibulargegend. Am 3I. desselben Monats in die hiesige chirurgische Klinik aufgenommen.

Status praesens: Von grazilem Knochenbau. Haut zart und leicht anämisch. Muskulatur etwas schwach entwickelt, subkutanes Fettgewebe ein wenig reduziert. Sichtbare Schleimhäute ziemlich blaß. Zunge feucht und ein wenig belegt. Puls regelmäßig, mäBig stark gespannt. Kopf normal geformt, dicht behaart, ohne Narben. Gesichtsausdruck nicht leidend. Augen und Ohren intakt. Nase ohne pathologischen Befund. Zähne gut entwickelt. Beide Tonsillen leicht angeschwollen. Thorax symmetrisch gebaut. Atemexkursionen beiderseits ohne Differenz. Leberlungengrenze liegt in der rechten Mammillarlinie am oberen Rande der 6. Rippe. Herz und Lungen ohne pathologischen Befund. Bauch weder aufgetrieben noch eingesunken, ohne abnorme Resistenz, nirgends druckempfindlich. Leberrand ca. $1 / 2 \mathrm{~cm}$ unterhalb des rechten Rippenbogens ziemlich hart fühlbar. Milz kaum zu fühlen, ebensowenig beide Nieren. Äußere Genitalien und Extremitäten intakt. Patellarsehnenreflex normal erhalten. Harn ganz klar, fast farblos, von saurer Reaktion, ohne pathologische Bestandteile.

Lokaler Befund: In der Frontalgegend nimmt man einen halbkugeligen, apfelgroßen, schwarz-rötlich verfärbten Tumor wahr, der ca. $7 \mathrm{~cm}$ breit und $3 \mathrm{~cm}$ hoch ist. Der Tumor ist auf der Unterlage verschieblich. Die Konsistenz desselben ist elastisch-weich am Zentralteil, etwar härter an der Peripherie. In den beiden Retromandibulargegenden finden sich taubeneigroße, elastisch-harte, glatte, nicht druckempfindliche Drüsenschwellungen, die Haut darüber rechts etwas rötlich verfärbt, links normal. Sie sind weder mit Unterlage noch mit Haut verwachsen. Kleinere Drüsenschwellungen von gleicher Beschaffenheit sind in der Submental-, Submaxillar- und Präaurikulargegend nachzuweisen. Die Wassermannsche Reaktion negativ.

Verlauf: 15. VI. Ein erbsengroßer und ein anderer bohnengroßer Tumor oberhalb des rechten Jochbogens aufgetreten. I7. VI. Mehrere erbsen- bis bohnengroße Drüsenschwellungen in der rechten Achselhöhle nachweisbar. I9. VI. In der linken Temporalgegend findet man eine bohnengroße Anschwellung. Die oben erwähnten Tumoren nehmen alle allmählich an Größe zu. 20. VI. Die rechten kubitalen, die linken axillaren, die beiden inguinalen und subinguinalen Drüsen erbsen- bis bohnengroß vergrößert. 26. VI. Milz überschreitet 
den linken Rippenbogen um I. cm, Konsistenz elastisch-hart, Oberfläche glatt. 28. VI. Leberunterrand ein Querfinger breit unterhalb des rechten Rippenbogens zu palpieren. I2. VII. Milzkuppe liegt in der Nabelhöhe, Leberunterrand zwei Querfinger breit unterhalb des rechten Rippenbogens. 16. VII. Der Tumor in der Frontalgegend, sowic dic retromandibularen Drüsenschwellungen durch $\mathrm{Ra}$. diumbehandlung stark verkleinert. 23. VII. Milz überschreitet die Mittellinie nach rechts um $2 \mathrm{~cm}$; ihr Unterrand liegt in der Höhe der Spina iliaca ant. sup. Gesicht und beide Unterextremitäten ödematös angeschwollen. Stimmung und Appetit sehr schlecht. 28. VII. Eine rechte Subkonjunktivalblutung aufgetreten. 29. VII. Heute geht das Kind unter zunehmendem Marasmus zugrunde.

Blutbefund:

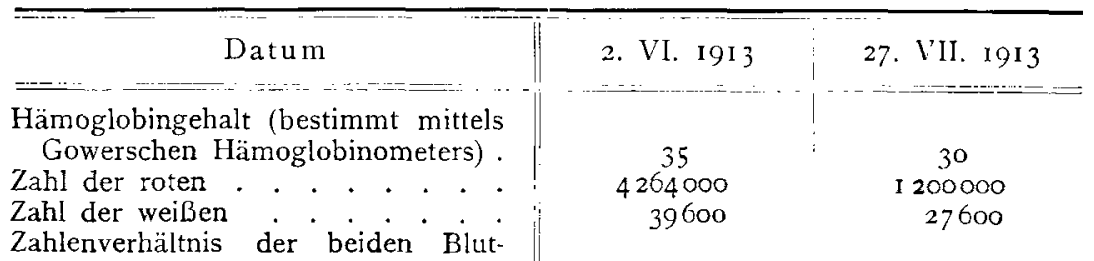
$\mathrm{W}: \mathrm{R}=\mathrm{I}: 108 \quad \mathrm{~W}: \mathrm{R}=\mathrm{I}: 43: 4$ Prozent. Verhältnis der WeiBen:

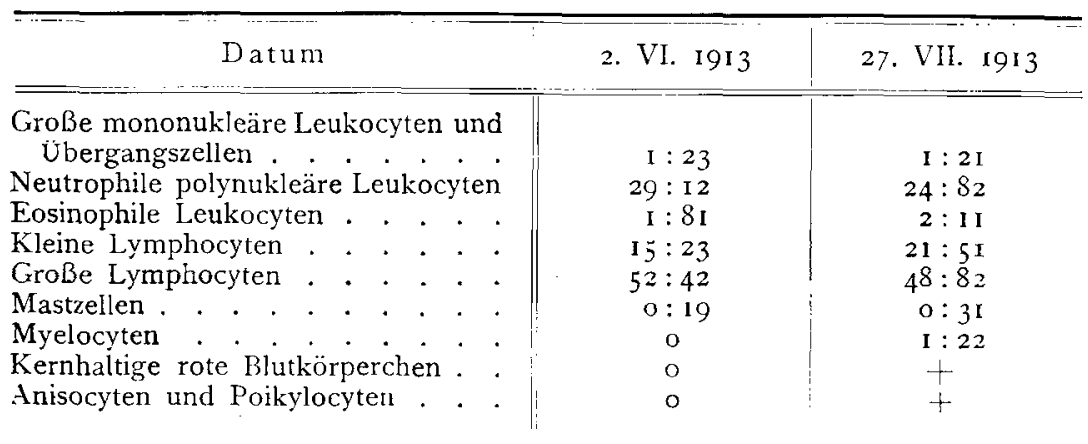

Obduktionsbefund:

Sektion am 30. VII. I9I3 (Dr. Kawakami): Aus dem Protokoll cntnehmen wir auszugsweise die folgenden Befunde: Körperlänge $90 \mathrm{~cm}$, Körpergewicht I $2 \mathrm{~kg}$. Ernährungszustand etwas schlecht. Livores am Rücken ziemlich stark ausgebildet. Haut im allgemeinen blaß. Eine rechte Subkonjunktivalblutung sichtbar. In der Stirngegend cine kindshandtellergroße, mit einer schwarz-bräunlich verfärbten Kruste bedeckte Stelle vorhanden. Beim Entfernen der 
Kruste nimmt man daselbst eine grau-weißlich belegte Granulationsfläche wahr, mit hier und da zerstreut liegenden unregelmäßigen Blutungsherden. In den beiden Retromandibulargegenden erbsengroße Granulationsflächen sichtbar. Abdomen stark aufgetrieben. Zwerchfell steht links an der 4. Rippe, rechts im 3. Interkostalraum. Milz reicht in der linken Mammillarlinie 4, in der linken Parasternallinie 5 Querfinger breit vom Rippenbogen abwärts. Leberrand liegt in der Medianlinie $\mathbf{I} \mathbf{1 , 2} \mathrm{cm}$ vom Proc. xyphoideus, in der rechten Mammillarlinie $3.5 \mathrm{~cm}$ vom Rippenbogen nach unten entfernt. Gedärme von mittlerer Weite. Serosa derselben glatt, glänzend. Omentum majus nach oben aufgerollt, fettarm. Serosa desselben glatt, glänzend. In der Bauchhöhle $50 \mathrm{ccm}$ gelber, klarer Flüssigkeit vorhanden. Pleurahöhlen: Pleura glatt, glänzend, keine Adhäsionen, links $50 \mathrm{ccm}$ bräunlich-rötlicher, klarer, rechts $100 \mathrm{ccm}$ rötlich-gelblicher, klarer Flüssigkeit enthalten. Herzbeutel: Einige Kubikzentimeter klarer Flüssigkeit darin. Herz ebenso groß wie Faust der Leiche, Konsistenz etwas weich. Venöse Ostien normal weit. Klappen ohne Veränderungen. Tonsillen beiderseits erbsengroß angeschwollen. Larynx und Trachea: Mukosa etwas getrübt, sonst nichts Besonderes. Ösophagus, Schilddrüse ohne pathologischen Befund, Lungen von mittlerem Volumen, Luftgehalt normal. Oberfläche glatt und glänzend, Konsistenz elastisch-weich. Schnittflächen grau-rötlich. Bronchien intakt. Milz I 8,5: I I:3,9 cm,groß; Gewicht 540 g. Serosa glatt, an der Oberfläche schwarz-rötliche, etwas erhabene, scharf abgegrenzte, landkartenartige Herde hier und da vorhanden. Schnittflächen uneben wegen der Aufschwellung der Follikel, Pulpa teigig weich, Trabekel deutlich entwickelt. Nebennieren ohne abnormen Befund. Nieren: Form und Konsistenz normal. Schnittflächen glatt, etwas getrübt, grau-rötliche keilförmige Herde hier und da sichtbar. Punktförmige hämorrhagische Herde im linken Nierenbecken vorhanden. Magen, Duodenum und Darm: Schleimhaut etwas angeschwollen, leicht getrübt, mit Schleim bedeckt. Ductus choledochus leicht durchgängig. Leber: Serosa glatt, glänzend, mit zahlreichen punktförmigen, hämorrhagischen Herden. Konsistenz elastisch-weich. An den Schnittflächen zahlreiche miliar- bis hanfkorngroße, scharf abgegrenzte grau-weißliche Knoten sichtbar. Venenwand im allgemeinen verdickt. In der Intima größerer Venen mehrere punktförmige, hämorrhagische Herde vorhanden. Ductus hepaticus und Gallenblase ohne pathologischen Befund. Pankreas normal groß, Serosa glatt, Schnittflächen etwas getrübt. Harnblase, Genitalien normal. Oberschenkelknochen: Knochenmark von grau-rötlicher Farbe. Compacta von normaler Beschaffenheit. Periost nicht verdickt. Beiderseitige Halsdrüsen erbsen- bis bohnengroß, Axillar- und Inguinaldrüsen bohnen- bis taubeneigro $B$ angeschwollen; Konsistenz elastisch-weich; Schnittflächen von grau-weißlicher Farbe, mit hier und da zerstreut 
liegenden größeren oder kleineren hämorrhagischen Herden. Bronchialdrüsen hanfkorn- bis erbsengroß angeschwollen, Schnittflächen anthrakotisch mit grauen, mäßig transparenten Inseln. Lymphdrüsen am Hilus der Milz und an der Porta hepatis erbsen- bis taubeneigroß, einige Mesenterial- und Retroperitonealdrüsen erbsengroß angeschwollen.

Mikroskopischer Befund:

a) Stirntumor. Die Epidermis in der Umgebung der Granulationsfläche im allgemeinen ziemlich dünn. Stratum germinativum besteht aus 5 bis 8 übereinander liegenden Zellreihen. In manchem Zellleib befindet sich eine große Vakuole. Der leicht geschrumpfte, runde oder abgeplattete Kern liegt bald zentral, bald ganz peripher. Im Stratum granulosum findet man 2 bis 3 Lagen von verschieden abgeplatteten Zellen. Körnchen in den stark abgeplatteten Zcllen fein, die in den weniger stark abgeplatteten gröber. Stratum corneum ziemlich dünn. Die Epidermis ist manchmal sehr dünn, sogar durchbrochen. Corium ist von zelligen Elementen, hauptsächlich von großen Lymphocyten und Erythrocyten infiltriert. Mitosen ziemlich reichlich. Die Infiltrate durchsetzen hier und da die ganze Epidermis und enthalten in sich zahlreiche neugebildete Kapillaren.

b) Lymphdrüsen. Die Abgrenzung der Follikel von Marksträngen ist infolge des sehr beträchtlichen Zellreichtums meist undeutlich. Das Gewebe ist von gleich großen mononukleären Zellen gleichmäßig durchsetzt. Mitosen und Riesenzellen ziemlich reichlich vorhanden. Hier und da sieht man nur Reticula und Blutgefäße. Man kann Sinus nicht gut erkennen. Die Drüsenkapsel nicht infiltriert.

c) Milz. Pulpa und Follikel nirgends deutlich differenziert. Das ganze histologische Bild stellt sich als eine ziemlich einförmige, ausgeprägt zellige Struktur dar. Auch die Oxydasereaktion zeigt einen durchaus negativen Ausfall. Unter den großen Zellformen findet man zerstreut liegende kleine Lymphocyten, welche an einigen Stellen sich mehr oder weniger anhäufen und so auf die Follikelbildung andeuten (s. Fig. I). Follikel sind manchmal durch die großen Lymphocyten total verdrängt, indem die letzteren kleine runde Herde bilden. Sehr oft liegen die großen Lymphocyten entlang den Blutgefäßen und durchsetzen die Wände derselben partiell bzw. total. Mitosen sind hier und da vorhanden, Ricsenzellen nirgends. An den Ausstrichpräparaten findet man meist große einkernige Zellen, daneben Erythrocyten, kleine Lymphocyten, neutrophile polynukleäre Leukocyten und Mastzellen in sehr geringer Zahl.

d) Leber. In der Umgebung der Gefäße, im interazinösen Bindegewebe finden sich große, unregelmäßig gestaltete, zellreiche Herde, die vorwiegend aus großen, einkernigen Zellen bestehen, nebst einer 
ganz geringen Anzahl von kleinen Lymphocyten (s. Fig. 2). Diese Herde verdrängen bzw. substituieren einige Läppchen total oder partiell. In ihnen sind keine bindegewebigen Gerüste nachzuweisen, wohl aber Gitterfasern, welche aus Gefäßwänden entspringen und sich zwischen einzelnen großen Lymphocyten bzw. Haufen derselben dendritisch verzweigen. Kapillaren sind mit großen Lymphocyten ziemlich ungleichmäßig gefüllt: bald in ihrer ganzen Ausdehnung, bald nur peripher, bald nur zentral. Die mit denselben nicht gefüllten Teile der Kapillaren sind durch Blutstauung stark erweitert. Mitosen kommen in den Herden spärlich vor, Riesenzellen nirgends.

e) Nieren. Die lymphoiden Herde liegen hier im perivaskulären Gewebe, in der Umgebung des Glomerulus, oder zwischen einzelnen Harnkanälchen. Einige Glomeruli sind von den Lymphoidzellen total durchsetzt. Diese Zellen infiltrieren auch Gefäßwände teilweise oder gänzlich. Mitosen sind in den Herden ganz spärlich wahrnehmbar.

f) Knochenmark. An den Ausstrichpräparaten erweist sich dasselbe als sehr zellreich. Die großen einkernigen Lymphocyten machen $2 / 3$ der gesamten Zellen aus. Das übrige Drittel kommt den Erythrocyten. Hormoblasten, Myelocyten, Übergangszellen und eosinophilen Zellen zu.

g) Verhalten der lymphoiden Herde zu den Gefäßen. Die lymphoiden Herde finden sich perivaskulär in den fast sämtlichen oben erwähnten Organen, wenigstens ihre Andeutungen. Sie durchsetzen Gefäßwände bald total, bald nur partiell (s. Fig. 3). So findet man einige $G$ efäße, bei denen nur Adventitia oder Adventitia und Media infiltriert sind. Bei anderen sind die Zellen bereits in die Intima eingedrungen, wo sie bald gleich-, bald ungleichmäßige Verdickungen bilden und dadurch das Endothel ins Lumen hineintreiben. Die total infiltrierten Gefäße haben ein so stark verengtes und exzentrisch verlagertes Lumen, daß man sie nur durch den Nachweis elastischer Fasern erkennen kann, die in der Regel mehr oder weniger gut erhalten geblieben sind. An mancher Stelle nimmt man eine Kontinuitätstrennung der Elastica interna, sowie der Intima und des Endothels wahr, durch welche die Zellen ins Lumen gelangen können. Die Gefäße sind meist leer. Nur bei wenigen finden sich im Lumen lymphoide Zellen zu größeren oder kleineren Haufen zusammengelagert neben gut erhaltenen roten Blutkörperchen.

Banti faßt, wie eingangs erwähnt, die Leukämie als ein Sarkom auf. Die lymphoiden Zellen, welche die Wände der Gefäße in den leukämisch erkrankten Organen durchbrechen und in die entfernt liegenden Organe gelangen, sollen die Fähigkeit weiterer Entwicklung besitzen, selbst wenn daselbst normalerweise kein lymphatisches Gewebe vorhanden ist. Babes beschreibt 
eine neoplastische Leukämie. Im Blute fand er deformierte rote Blutkörperchen, Lymphocyten und polynukleäre Leukocyten (kaum ıo Proz. der zelligen Elemente) und ovale Zellen mit großen, länglichen, oft blasigen Kernen (etwa 90 Proz.). Es handelte sich dabei wohl um eine Neoplasie endothelialer Herkunft, eine seltsame Abart der Leukämie. Ogawa berichtet über einen Fall akuter lymphatischer Leukämie, entstanden im Anschluß an ein primäres Thymussarkom. Die hämatologischen Befunde desselben zeichneten sich durch eine starke Verminderung der roten Blutkörperchen bis auf I 258500 und durch eine Zunahme der weißen bis auf 108000 aus, 90 Proz. der letzteren waren kleine Lymphocyten. Buschke und H. Hirschfeld sahen bei einem Fall in Blutbildungs-, sowie in anderen Organen hier und da perivaskuläre Rundzellenanhäufungen; ein Hineinwachsen der Geschwülste in die Blutgefäße war nicht festzustellen. Sie behaupten, daß die Tumorzellen wahrscheinlich primär im perivaskulären Gewebe wucherten und daß die Weiterverbreitung derselben von dort aus offenbar auf dem Wege der Blutbahn erfolgte. Kundrat und Paltauf fassen gewisse Erkrankungen des lymphatischen Apparates, charakterisiert durch einen besonderen histologischen Befund, sowie durch eine eigentümliche Art von Ausbreitung und Verlauf, worauf wir später zurückkommen werden, als die Lymphosarkomatose zusammen. Die Leukosarkomatose Sternbergs soll sich durch das Vorhandensein einer Geschwulstbildung nach Art des Lymphosarkoms und durch das hämatologische Bild der akuten Leukämie mit Auftreten zahlreicher großer einkerniger Lymphocyten (im Maximum 99 Proz., im Minimum 45-50 Proz.) charakterisieren.

Gehört unser Fall zur Leukosarkomatose? Außer, daß bei demselben große Lymphocyten etwas weniger vorhanden waren $(48,82-52,42$ Proz.), stimmen die Verminderung der roten Blutkörperchen (bis auf I 200000 ) und der mikroskopische Befund des Knochenmarks, sowie der Organe, wie Milz, Leber, Nieren usw., im großen und ganzen mit den Angaben Sternbergs über die atypische Wucherung des lymphatischen Apparates überein. Es liegt hier eine atypische, großzellige Wucherung des lymphadenoiden Gewebes mit leukämischem Blute vor, die S ter nberg von der echten kleinzelligen lymphatischen Leukämie, von der ein- 
fachen Hyperplasie des lymphatischen Gewebes getrennt wissen will. Die großzellige Leukämie steht nach ihm den Lymphosarkomen näher, von denen sie sich nur durch den Blutbefund und das Befallensein von Milz und Knochenmark unterscheidet. Er sieht eben in den großen Lymphoidzellen keine typischen, Lymphocyten, obschon sie mit denselben den Entstehungsort gemeinsam haben; er vindiziert ihnen vielmehr den Charakter von Geschwulstzellen.

Kann die echte kleinzellige, rein hyperplastische, lymphatische Leukämie auf die Ungebung übergreifende, atypische, mit heterotopen Bildungen einhergehende Wucherungen des lymphatischen und hämatopoëtischen Apparates niemals erzeugen? Fabian, Naegeli und Schatiloff untersuchten acht Fälle kleinzelliger lymphatischer Leukämie, fanden in fast allen ein mehr oder weniger aggressives Wachstum und kamen zu dem Schlusse, daß sich eine scharfe Grenze nicht ziehen lasse, zwischen der kleinzelligen, lymphatischen Leukämie und der Leukosarkomatose, indem sie nur zwei graduell verschiedene Prozesse darstellen. Beim Fall 8 von V. Müllern-Großmann mit einer großen Geschwulstmasse im Mediastinum fand man im Blute nahezu ausschließlich kleine Lymphocyten. Andererseits wurde der vorwiegend großzellige Blutbef und trotz Fehlens einer sarkomartigen Tumorbildung mehrfach beschrieben. So berichten Meyer und Heineke über eine akut verlaufende einfache hyperplastische Leukämie, bei der sich fast 90 Proz. große Lymphocyten im Blut fanden. Ähnliche Mitteilungen wurden von Lucksch-Pietrowski, O. Moritz, Pappenheim. Hirschfeld und Graetz gemacht. Der letzte fand ferner in einem Fall chronischer, kleinzelliger, lymphatischer Leukämie eine sehr ausgedehnte Infiitration der Drüsenkapsel, sowie des Fett- und Bindegewebes, die an manchen Stellen einen recht erheblichen Grad erreichte. Somit sollen nach ihm die von Sternberg für die großzellige Leukämie als charakteristisch angegebenen Kriterien in gleicher Weise für die kleinzellige lymphatische Leukämie gelten. Türck konstatierte bei einer typischen chronischen, kleinzelligen, lymphatischen Leukämie ein Lymphosarkom in einer mesenterialen Lymphdrüse, während er bei mehreren großzelligen Leukämien nirgends lymphosarkomatöse 
Neubildungen fand. Freilich gibt Sternberg selbst die Möglichkeit $z u$, daß Übergänge zwischen der lymphatischen Leukämie und der Leukosarkomatose existieren können. Nach Pappenheim existiert wohl eine Leukosarkomatose, doch sei dieselbe keine Erkrankung des lymphatischen Apparates sui generis mit ganz eigentümlichen Lymphocyten. Herz ist der Ansicht, daß gewisse Beziehungen zwischen der leukämischen Hyperplasie einerseits und der Lymphosarkomatose andererseits bestehen können. Einen Beweis dafür, daß die lymphoiden Zellen durch Zerstörung der Gefäßwandung direkt ins Blut eindringen können, suchte Banti in einem seiner Fälle dadurch zu führen, daß die weißen zirkulierenden Zellen plötzlich eine starke Vermehrung zeigten (von 23000 auf 280000). Zugleich traten eine beträchtliche Menge von Gefäßendothelien im Blut auf, die am folgenden Tage fast ganz verschwanden. Graetz fand bei einer großzelligen lymphatischen Leukämie eine hochgradige Anhäufung lymphatischen Gewebes entlang größeren Gefäßstämmen in der Leber. Einige Autoren (B e nda, Mosse) führen die Entstehung der leukämischen Blutveränderung auf die Lymphocyteninfiltration in der Wand kleiner Blutgefäße in den leukämischen Lymphomen zurück. Nach Paltauf und Sternberg führt eine lokale geschwulstartige Gewebsproliferation sekundär zur leukämieartigen Blutveränderung.

Bei unserem Fall vergrößerten sich die rechten kubitalen, die linken axillaren, die beiden inguinalen und subinguinalen Drüsen auf einmal. Man könnte all diese Drüsenschwellungen als echte Metastasen durch Lymphbahnen oder, wie es Banti auffaßt, als sekundäre Metastasen durch Blutbahnen nach Durchbruch der Gefäßwand betrachten. Wir halten sie vielmehr für autochthone Wucherungen, die unter dem Einflusse der überall im Organismus kreisenden Noxe an Ort und Stelle entstanden waren. Ein und dieselbe Noxe bewirkt je nach dem Reaktionsvermögen der Gewebe hier nur eine hyperplastische, dort eine tumoröse Wucherung, und beidemal wird das Blut symptomatisch leukämisch, sobald die Gefäßwand irgendwo durchwachsen wird. Die hyperplastische und die tumoröse Wucherung des lymphatischen und hämatopoëtischen Apparates sind sicherlich verschiedene Manifestationen ein und desselben Prozesses. Wie aus Berichten man- 
cher Autoren und aus unserer Beobachtung ersichtlich, können die lymphatischen Zellen nach Durchbruch der Gefäßwand in lymphatischen Herden direkt ins Blut eintreten.

Die lymphatische Leukämie gehört unserer Ansicht nach nicht in die Kategorie der malignen Tumoren, indem sie eine Systemerkrankung der lymphatischen und hämatopoëtischen Organe darstellt. Der Blutbefund hat sonach nur eine symptomatische Bedeutung. Ferner sind wir geneigt, zwischen der großund der kleinzelligen Leukämie keine scharfe Trennung zu machen, vielmehr eine „,innige Familienzugehörigkeit" dieser beiden Arten der Leukämie mit einem graduellen Unterschiede anzunehmen.

Wie bezieht sich die Leukämie auf die aleukämische Lymphomatose? Ribbert ist der Ansicht, daß die lymphatische Leukämie nur eine Variation der aleukämischen Lymphomatose sei. Baumgarten bemerkt: „Pseudoleukämie ist eine Form (eine Unterart oder Modifikation) der chronischen Iymphatischen Leukämie.“ Borst sagt, ,die enge Beziehung der Leukämie und Pseudoleukämie wird durch neuere Untersuchungen immer mehr anerkannt". Pappenheim betont die histologische Gleichheit der lymphoiden Pseudoleukämie und Leukämie. Nach Hirschfeld und Troje stellt die aleukämische Lymphomatose ein Vorstadium der Leukämie dar. In neuerer Zeit erzielten Ellermann und Bang durch die Impfung des an Pseudoleukämie gestorbenen Hühnern entnommenen Materials bald Leukämie, bald Pseudoleukämie. Die Ansicht von Domarus ist wie folgt: wenn es gleichzeitig zu einer Ausschwemmung der gewucherten Zellen ins Blut kommt, so hat man klinisch eine echte Leukämie vor sich, im anderen Fall ist es eine sogenannte Aleukämie, die von der ersteren nur durch ein untergeordnetes, nicht wesentliches Symptom, das Fehlen der Blutveränderungen, verschieden ist und jederzeit in die echte Leukämie überzugehen vermag.

Wie bereits erwähnt, sahen wir in unserem Fall die lymphoiden Zellen Gefäßwände in den Herden der untersuchten Organe immer von außen nach innen hin durchwachsen. Wenn dieselben endlich ins Blut eintreten, wie es bei unserem Fall an mancher Stelle der Fall war, so entsteht ohne Zweifel die typische lymphatische Leukämie; solange der Durchbruch der Gefäßwände 
nicht stattfindet, hat man die Pseudoleukämie oder aleukämische Lymphomatose. Die aleukämische Lymphomatose ist demnach eine Unterart oder das Anfangsstadium der lymphatischen Leukämie, indem zwischen den beiden nur ein quantitativer, kein qualitativer Unterschied besteht.

Wie verhält sich Sternbergs Leukosarkomatose mit der Kundrat-Palta ufschen Lymphosarkomatose? Das Charakteristikum der Leukosarkomatose besteht nach Sternberg darin, daß eine zunächst lokale geschwulstartige Gewebsproliferation sekundär zur leukämieartigen Blutveränderung führt. Die Lymphosarkomatose stellt sich nach $\mathrm{K} u n d \mathrm{r}$ at als eine von einer Gruppe von Lymphdrüsen oder Lymphfollikeln ausgehende Geschwulstbildung ohne Blutveränderung dar. Sternberg will die beiden Erkrankungen durch den Blutbefund, sowie durch die Ausbreitung des Prozesses voneinander unterscheiden. Bei der letzteren bestehe keine wesentliche Vermehrung der Lymphocyten bzw. der großen einkernigen Leukocyten im Blut, während gerade die Vermehrung der letzterwähnten Zellart ein charakteristisches Merkmal der ersteren darstelle. Ferner komme der Lymphosarkomatose die universelle Ausbreitung über das gesamte lymphatische System nicht zu. Die Fälle von Lymphosarkomatose, die mit einer beträchtlichen Vermehrung großer einkerniger Leukocyten im Blut einhergehen, soll nach ihm der Leukosarkomatose zugehören. Die Blutgefäße in der Lymphosarkomatose, die von der Geschwulst umschlossen waren, fand Kundrat meist komprimiert, meist obliteriert, niemals durchbrochen. Der oben erwähnte Fall Leukosarkomatose von Buschke und H. Hirschfeld zeigte anfangs für eine längere Zeit den normalen Blutbefund; erst spät wurden die Zellen des Tumors im Blut gefunden.

Die Leukosarkomatose kann somit zustande kommen, wenn die lymphoiden Zellen der Lymphosarkomatose nach Durchbruch der Gefäßwand ins Gefäßlumen gelangen. Demnach nehmen wir an, daß zwischen den beiden Erkrankungen keine scharfe Trennung zu machen ist, indem auch hier eine, innige Familienzusammengehörigkeit" mit einem mehr graduellen Unterschiede besteht.

Wie entsteht die leukämische Blutveränderung? Ehrlich 
macht einen prinzipiellen Unterschied zwischen der lymphatischen und der gemischtzelligen Leukämie. Bei der ersteren finde eine passive Ausschwemmung, d. h. eine Ausspülung der in gesamten lymphatischen und hämatopoëtischen Apparaten an verschiedenen Stellen des Körpers in überaus reichlicher Menge gebildeten Lymphocyten in die Blutbahn statt. Bei der letzteren komme hingegen eine aktive Auswanderung der Myelocyten aus dem Knochenmark in die Blutbahn zustande; es sollen dabei unter dem Einfluß der spezifisch leukämischen Noxe nicht nur die fertigen polynukleären Elemente, sondern auch ihre mononukleären, eosinophilen, sowie neutrophilen Vorstufen ins Blut einwandern. Auf welche Weise die Ausschwemmung der Lymphocyten in den Fällen lymphatischer Leukämie sich vollzieht, ist zurzeit noch nicht ganz geklärt. Neumann, Walz, Pappenheim u. a. behaupten, daß alle Leukämien ihre Entstehung dem Knochenmark verdanken. Bei der myeloiden Leukämie handele es sich um eine Hyperplasie des unveränderten Knochenmarks, bei der lymphatischen hingegen um eine Hyperplasie des lymphoid ausgearteten. Wenn die formative Reizung Milz und Lymphdrüsen treffe, häufen sich die neugebildeten Lymphocyten in diesen Organen an, die infolge der Dehnbarkeit der Kapseln sich vergrößern: das ist die Pseudoleukämie. Ergreife sie das Knochenmark, so kann sich das Knochengewebe nicht ausdehnen; die Lymphocyten treten ins Blut und bewirken die Leukämie.

Wie bereits erwähnt, fanden wir in unserem Fall nicht nur die Wände kleiner Blutgefäße in den leukämischen Lymphomen, sondern auch die größerer in der Nähe der lymphoiden Herde von lymphoiden Zellen durchsetzt, die hier und da ins Gefäßlumen gelangten. Somit ist unserer Ansicht nach die Lymphocytenausschwemmung nicht eine Steigerung eines physiologischen Vorgangs, wie v. Schuhmacher behauptet, sondern die Folge einer pathologischen Wucherung unter dem Einfluß der spezifisch leukämischen Noxe.

Wie ist das Schicksal der ins Blut ausgeschwemmten Lymphocyten? Bei der Blutuntersuchung fanden wir, daß sich Hand in Hand mit Vermehrung der Lymphocyten freie Kerne und Kernzerfallsformen im Blutt vermehrten. Die letzteren sind ohne Zweifel Zerfallsprodukte der Lymphocyten und werden selbst 
endlich spurlos aufgelöst. Bei einer akuten Vermehrung der weißen Blutzellen findet man klinisch meist eine mehr oder weniger hohe Körpertemperatursteigerung, welche wohl als Reaktionsfieber gegen die Zerfallsprodukte der pathologisch ausgeschwemmten Lymphocyten anzusehen ist.

In welcher. Beziehung steht die Stembergsche Leukosarkomatose zur Myeloblastenleukämie? Diese beiden Erkrankungen haben eine große Ähnlichkeit miteinander, sowohl klinisch, als auch hämatologisch und histologisch. Es ist wohl sicher, daß es sich bei einer Mehrzahl der von Ebstein, Fraenkel und anderen als die akute lymphatische Leukämie veröffentlichten Fälle um die Myeloblastenleukämie handelte. Theoretisch muß es zwei Formen der akuten Leukämie geben, die genau den bekannten chronischen Formen entsprechen: die akute lymphatische Leukämie und die Myeloblastenleukämie. Man legt bei ihrer Unterscheidung einen größeren Wert auf den Organbefund als auf den Blutbefund. Viele Autoren sind der Ansicht, daB bei der Myeloblastenleukämie die Atrophie der Follikel infolge der myeloiden Umwandlung vorliege, während bei der lymphatischen die Hypertrophie derselben nachweisbar sei. Pappenheim sieht hingegen die interfollikuläre Veränderung als primär an. Die Unterscheidung der beiden Formen ist fast unmöglich, wenn die ganze Drüse von den morphologisch nicht deutlich unterscheidbaren großen mononukleären Zellen ohne scharfe Grenze zwischen follikulären und interfollikulären Geweben dicht und diffus infiltriert ist. Wenn durch die rapide Wucherung des myeloiden Systems ganz unreife granulafreie große und kleine Zellen, Myeloblasten, massenhaft in die Zirkulation gelangen, dann wird das Blutbild dem einer lymphatischen Leukämie in hohem Grade ähnlich, und die Differentialdiagnose ist nach Fabian, Naegeli und Schatiloff hämatologisch nur möglich: I . durch die Altmann-Schriddesche Färbung, 2. durch den Nachweis von 4-6 Nukleolen bei Methylgrün-Pyronin-Färbung, 3. durch das Vorkommen aller $Z$ wischenstufen zwischen Myeloblasten und Myelocyten, 4. durch die große und nicht rasch sinkende Zahl anderer myeloischer Elemente, namentlich regelrechter eosinophiler und neutrophiler Nyelocyten, 5. durch das Vorkommen geradezu massenhafter kernhaltiger roter Blutkörperchen in 
manchen Fällen und 6. durch den Nachweis von Knochenmarksriesenzellen.

Das Blutbild unseres Falles war durch eine sehr beträchtliche relative und absolute Vermehrung großer, einkerniger Zellformen charakterisiert. Diese großen Zellformen waren $\mathrm{I} 2$ bis $20 \mu$ groß. Ihre Kerne waren äußerst blaß, chromatinarm, rund oder oval, enthielten I-4 deutliche Nukleolen, zeigten oft eine fädignetzartige Struktur und waren manchmal an einer Seite mit einer Einbuchtung versehen (bis zur Hufeisenform). Das Protoplasma war verschieden stark entwickelt und bildete einen breiteren oder schmäleren Saum um die Kerne. Dasselbe war stark basophil, zeigte bei Triazidfärbung keine Granula, bei Färbung nach MayGrünwald mehrere undeutliche, blaue Körnchen. Diese Zellformen sind bisher nicht einheitlich gedeutet und verschieden bezeichnet: so große Lymphocyten (Ehrlich und Fränkel), indifferente Lymphoidzellen (Michaelis, Wolff), Stammzellen (T ürk), Lymphoblasten (Ka u f mann), unreife Zellen (E. Grawitz), Lymphogonien (Benda), Markzellen (Troje), Céllules médullaires (Cornil), Hämo- und Lymphomakrophagen (Metschnik off), Myeloblasten (Naegeli) usw.

Myeloblasten und große Lymphocyten voneinander zu unterscheiden, ist in ungefärbten Präparaten unmöglich, wie P a p penheim und Butterfield bemerken. Bei starker Hitzefixation und Methylenblaufärbung sollen sich nach $\mathrm{Naegeli}$ Lymphocytenkerne ganz blaß färben, mit I-2 sehr schönen Nukleolen, Myeloblastenkerne hingegen helldunkelblau, mit 2-4 ganz undeutlichen Kernkörperchen. Das Pyroninmethylgrün soll 3 bis 4 Nukleolen im Myeloblasten- und $\mathrm{I}-2$ im Lymphocytenkern rot färben.

Die Angabe von $\mathrm{Na}$ a geli konnten wir nicht bestätigen, da wir, wie bereits erwähnt, im Kerne dieser großen Zellformen bald I, bald 2--3 Nukleolen fanden, so daß kein Unterschied bezüglich der Zahl derselben zwischen den beiden Arten von Zellen zu konstatieren war. Die morphologischen Unterschiede, wie von Schridde erwähnt, sind nach unserem Ermessen sehr unbedeutend: Die Größe von den beiderlei Zellen fast gleich, ebenso die äußere Konfiguration der Kerne, das Protoplasma gleich basophil. Nur insofern differieren die Lymphoblasten von den Myelo- 
blasten, daß die ersteren einen schmäleren hellen Hof um den Kern besitzen, als die letzteren. Ein weiterer Unterschied besteht nach Winkler darin, daß nur die Ehrlichschen Granula die Oxydasereaktion geben, $d$. h. sich bei der Behandlung mit $\alpha$ Naphthol und Dimethyl-p-Phenylendiamin blau färben. Wir möchten unsererseits diese großen Zellformen ,große Lymphocyten" nennen, da das von denselben infiltrierte Milzgewebe nach Schultze ungefärbt blieb, während das leukocytäre und myeloische Gewebe sich blau färbte. Anderersteits betonen Jochmann, Blühdorn, Laederich, Debri und Gastinel, daß die Oxydase und das proteolytische Ferment in den Myeloblasten manchmal fehlen können. Klieneberger fand im Blut einer mit Röntgenstrahlen behandelten chronischen Myelämie einkernige ungranulierte Zellen ohne die Oxydasenreaktion. Dessenungeachtet zählt er die Zellen zu đen Myeloblasten und bringt die Myeloblastenausschwemmung mit der Bestrahlungstherapie in Zusammenhang. Nach Jagič und Neukirch treten im Blut von den mit Röntgenstrahlen behandelten Fällen der chronischen myeloischen Leukämie neben den typischen Myeloblasten große ungranulierte, mononukleäre Zellen von $\mathrm{Naegeli}$ auf, die die Kriterien der Myeloblasten, wie Oxydasenreaktion, Basophilie des Protoplasmas usw. nicht aufweisen sollen. Wolff nimmt an, daß die indifferenten Lymphoidzellen einer Weiterestwicklung zu Lympho- oder Myelocyten fähig seien und daß sich die Lymphoidzellenleukämie im weiteren Verlaufe in eine rein lymphatische bzw. myelogene Leukämie umwandeln könne. Nach Pappenheim stimmen die Leukosarkomzellen von Sternberg mit seinen als gemeinsame Vorstufen der Lympho- und Myelocyten bezeichneten Stammzellen ganz überein, so daß die Fälle, welche Sternberg zur Leukosarkomatose zählt, cytologisch sowie lymphatisch zur myeloischen Leukämie gehören sollen. Paltauf sagt, daß die Tumorzellen Sternbergs keine buchtkernigen atypischen Riederformen, sondern vielmehr typische, lymphocytforme, makrolymphocytoide Lymphocyten (Myeloblasten, Großlymphocyten) seien. Alles in allem zweifeln wir selbst daran gar nicht, daß die Leukosarkomatose Sternbergs in einer innigen Beziehung zur Myeloblastenleukämie steht. 


\section{Zusammenfassung.}

I. Es handelte sich bei unserer Beobachtung um eine akute großzellige lymphatische Leukämie. Hervorzuheben sind dabei folgende Befunde:

a) Die primäre, apfelgroße, lymphoide Tumorbildung in der Stirngegend, entstanden im Anschluß an ein leichtes Trauma.

b) Zahlreiche Mitosen im Stirntumor, sowie in Lymphdrüsen und Infiltrationsherden in einigen Unterleibsorganen, wie Leber, Milz und Nieren.

c) Die außerordentlich starke Vermehrung der großen Lymphocyten im Blut.

d) Die lymphoiden Herde immer nachweisbar in der Umgebung der Gefäße in den oben erwähnten Organen.

e) Die lymphoiden Zellen infiltrierten zunächst nur Adventitia oder Adventitia und Media. Schließlich drangen sie in die Intima ein, woselbst manchmal das Endothel fehlte, so daß die lymphoiden Elemente frei ins Gefäßlumen eintreten konnten.

2. Wenn die lymphatischen Zellen Gefäßwände durchwachsen und endlich ins Blut eintreten, so entsteht die typische lymphatische Leukämie; solange der Durchbruch der Gefäßwände nicht stattfindet, hat man die Pseudoleukämie oder aleukämische Lymphomatose.

3. Die aleukämische Lymphomatose ist demnach eine Unterart oder das Anfangsstadium der lymphatischen Leukämie, indem zwischen Leukämie und aleukämischer Lymphomatose nur ein quantitativer, kein qualitativer Unterschied besteht.

4. Die tumoröse Wucherung bei der Leukämie entwickelt sich wohl unter dem Einfluß der spezifisch leukämischen Noxe und gehört nicht in die Kategorie der malignen Geschwülste.

5. Zwischen Sternbergs Leukosarkomatose und $\mathrm{K}$ undrat Paltaufs Lymphosarkomatose besteht eine ,innige Familienzusammengehörigkeit", mit einem mehr graduellen Unterschiede.

6. Die leukämische Blutveränderung ist durch die Lymphocytenausschwemmung infolge einer pathologischen Wucherung unter dem Einfluß der spezifisch leukämischen Noxe bedingt. Die ausgeschwemmten Lymphocyten werden im Blute endlich spurlos aufgelöst. 
7. Die Sternbergsche Leukosarkomatose steht zur Myeloblastenleukämie in einer innigen Beziehung.

\section{Literaturverzeichnis.}

I. B a bes, Neoplastische Leukämie. Zentralbl. f. allgem. Pathol. u. pathol. Anat. 1902, Bd. 13, S. 695.

2. Banti, G., Die Leukämien. Ebendaselbst 1904, Bd. I5, S. I.

3. Bardeleben-Bartels, Handbuch der Anatomie des Menschen. Das Lymphgefäßsystem. Jena 1909 .

4. Barrenschen, K. Hermann, Zur Frage der akuten Leukämie. Wiener klin. Wochenschr. 1912, Nr. 8, S. 293.

5. Borst, Max, Die Lehre von den Geschwülsten. Wiesbaden 1902.

6. B us chke, A., u. H i r c h f eld, Hans, Über Leucosarcomatosis cutis. Folia haematologica 19I I, Bd. I2, Teil 1, S. 73.

7. Domarus, V., Uber die Beziehungen der Leukämien zu den malignen Neoplasmen. Ebendaselbst 1912, Bd. I3, Teil I, S. 384.

8. Ellermann, V., น. B ang, O., Experimentelle Leukämie bei Hühnern. Zentralbl. f. Bakteriologie, Parasitenkunde u. Infektionskrankheiten I9o8, Originale, Bd. 46, H. 9, S. 595.

9. Fabian, E., Na e geli, O., U. Sch a t i l off, P., Beiträge zur Kenntnis der Leukämie. Virchows Arch. f. pathol. Anat. u. Physiol. u. f. klin. Med. 1907, Bd. 190, S. 436.

ıo. Fraenke1, Eugen, u. $\mathrm{M} \mathrm{u} \mathrm{ch,} \mathrm{Hans,} \mathrm{Bemerkungen} \mathrm{zur} \mathrm{Ätiologie}$ der Hodgkinschen Krankheit und Leukaemia lymphatica. Münchn. med. Wochenschr. I9Iо, Nr. I3, S. 685.

I I. F u j in a m i u. I n a moto, Über die sarkomatöse Neubildung der Hühner. Gann (japanisch) r9 Io, Jahrg. 3, S. 462.

I2. Goldmann, Dic Wandveränderungen an den Gefäßen im Bereiche der Neubildung und ihrer Umgebung. Deutsche med. Wochenschr. I906, Nr, 4l, S. 1683 .

13. Gorja ew, N., Zur Leukämiefrage. Folia haematologica I9I3, Bd. I6, Teil I, S. I 20.

I4. Gra etz, Fr., Uber lymphatische Leukämie, mit besonderer Berücksichtigung ihrer großzelligen Form. (Zugleich ein Beitrag zur Frage der Leukosarkomatose.) Zieglers Beitr. z. pathol. Anat. I9I0, Bd. 49, S. 338.

15. Grawitz, E., Das Wesen des leukämischen Krankheitsprozesses und die therapeutische Beeinflussung desselben. Berliner klin. Wochenschr. I908, Nr. 24, S. II 30 .

I6. H e rbst, Oskar, Leukämie mit Schädeltumoren bei einem einjährigen Kinde. Monatsschr. f. Kinderheilk. 1910, Bd. 9, S. 447.

17. H c rxhe imer, G., Über die Lymphoblasten- (großzellig lymphatische) und Myeloblastenleukämie. Münchn. med. Wochenschr. I913, Nr. 45, S. 2506 u. Nr. 46, S. 2573. 
I8. H e rz, Albert, Über die den Leukämien verwandten Krankheitsprozesse. Folia haematologica I912, Bd. I3, Teil I, S. 408.

19. H esse, Friedrich Adolf, Ein Beitrag zur Diagnostik der aleukämischen Lymphomatose (Lymphosarkomatosen). v. Bruns' Beitr. z. klin. Chir. 1912, Bd. 79 , S. 95 .

20. Hirschfeld, Hans, Über akute Leukämie. Folia haematologica 1907, Jahrg. 4, S. 202.

21. H ochstetter, F., Ein Fall von lymphatischer Leukämie. Ebendaselbst I912, Bd. I4, Teil I, S. 6r.

22. J a gi č, N. V., u. N e ukj j ch, P., Über das Auftreten großer mononukleärer ungranulierter Zellen im Blute chronischer Myelämien. Berliner klin. Wochenschr. I9ıo, Nr. I9, S. 874 .

23. Kelling, Georg, Über perniziöse Anämie und Leukämie. Münchn. med. Wochenschr. I906, Nr. 39, S. I90I.

24. Kos low ski, P. J., Zur Frage von bösartigem Granulum Benda. Russki Wratsch 19I2, Nr. II u. 12. Ref. in Zentralbl. f. Chir. I9I2, Nr. 23, S. 783 .

25. K u n drat, Über Lymphosarkomatosis. Wiener klin. Wochenschr. I893, Nr. I2, S. 2 I I.

26. Lucksch, Franz, Zur lymphatischen Leukämie. Folia haematologica rgo6, Jahrg. 3, S. 325 .

27. Moritz, Oswald, Zur Frage der akuten Lymphocytenleukämie und Pseudoleukämie. Ebendaselbst 1907, Jahrg. 4, S. 627.

28. M u ch, Hans, Über die granuläre, nach Ziehl nicht färbbare Form des Tuberkulosevirus. Beitr. z. Klinik d. Tuberkulose 1907, Bd, 8, S. 85.

29. - Über die Hodgkinsche Krankheit und ihre Beziehungen zur Tuberkulose. Ebendaselbst 1913, Supplementband 4, S. I95.

30. O gawa, Ein Fall akuter lymphatischer Leukämie, entwickelt auf der Basis von primärem Thymussarkom. Kyoto Igaku Zassi (japanisch) I905, Bd. 2, H. 3, S. 29.

3I. Palta uf, Rich., Leukosarkomatose und Myeloblastenleukämie. Wiener klin. Wochenschr. I912, Nr. I, S. 46.

32. Pappenheim, A., Über die Stellung der akuten großzellig-lymphocytären Leukämie im nosologischen System der Leukämien und die Bedeutung der großen Lymphocyten Ehrlichs an und für sich und für die Pathologie dieser Erkrankung. Folia haematologica I907, Jahrg. 4, S. I, I4I U. 535.

33. - Über zwei Formen akuter großlymphocytöser Leukämie. Zentralbl. f. allgem. Pathol. u. pathol. Anat. I907, Nr. 20, S. 823.

34. - Uber dei Anwendung des kombinierten May-Giemsa-Verfahrens zur Schnittfärbung. Folia haematologica I9II, Bd. II, Teil I, S. 373.

35. - Bemerkungen zur Frage der akuten Myeloblastenleukämie und Leukosarkomatose. Wiener klin. Wochenschr. I912, Nr. 4, S. I630.

36. Reschad, Hassen u. Schilling-Torga u, V., Über eine neue Leukämie durch echte Übergangsformen (Splenocytenleukämie) und ihre 
Bedeutung für die Selbständigkeit dieser Zellen. Münchn. med. Wochenschrift igr3, Nr. 36, S. I98r.

37. Ribbert, Hugo, Geschwulstlehre. Bonn 1904.

38. - Menschliche Zellen als Parasiten. Deutsche med. Wochenschr. 1907, Nr. 9, S. 329 .

39. Rodler-Zipkin, Rahel, Über einen Fall von akuter großzelliger lymphatischer Leukämie mit generalisierter Hauterkrankung. Virchows Arch. f. pathol. Anat. u. Physiol. u. f. klin. Med. I909, Bd. I97, S. 135. 40. Rosenfeld, Emst, Zur Lehre von der Hodgkinschen Krankheit. Berliner klin. Wochenschr. I9I I, Nr. 49, S, 2 I96.

4I. Ros enow, Georg, Klinische Beiträge zur Therapie der Leukämie mit Thorium X. Münchn. med. Wochenschr. I913, Nr. 40, S. 2214.

42. S a lmon, A., Über Lymphomatosen mit lokaler Aggressivität unter Beschreibung eines mit Hodentumor einhergehenden Falles. Deutsche med. Wochenschr. 1908, Nr. 10, S. 415.

43. Schultze, Walter, Ein Beitrag zur Kenntnis der akuten Leukämie. Zieglers Beitr. z. pathol. Anat. u. z. allgem. Pathol. 1906, Bd. 39, H. 2, S. 252 .

44. - Zur Differentialdiagnose der Leukämien. Münchn. med. Wochenschrift 1909, Nr. 4, S. 167.

45. Sternberg, Carl, Primärerkrankungen des lymphatischen und hämatopoëtischen Apparates; normale und pathologische Morphologie des Blutes. Ergebn. d. allgem. Pathol. u. pathol. Anat. I903, Jahrg. 9, Abt. 2, S. 360.

46. - Bemerkungen zu dem Aufsatze ,Zur lymphatischen Leukämie" von Dr. Franz L ucksch und zu der angefügten „Anmerkung des Herausgebers". Folia haematologica 1906, Jahrg. 3, S. 65 I.

47. - Über die akute myeloische Leukämie. Wiener klin. Wochenschr. 1911, Nr. 47, S. 1623 .

48. Troje, Uber Leukämie und Pseudoleukämie. Deutsche med. Wochenschrift i $892, \mathrm{Nr} .16$, S. 360.

49. Ts u noda, T., Über einen Fall von aleukämischem malignen Lymphom mit besonderen Leberveränderungen. Virchows Arch. f. pathol. Anat. u. Physiol. u. f. klin. Med. I9I I, Bd. 204, S. 265.

50. Türk, Wilhelm, Ein System der Lymphomatosen. Wiener klin. Wochenschr. I903, Nr. 39, S. 1073.

5I. - Meine bisherigen Erfahrungen über die Benzolbehandlung der Leukämien, verglichen mit den Ergebnissen anderer Methoden. Wiener med. Wochenschr. 19r3, Nr. Io, S. 626.

52. Veszprémi, D., Beiträge zur Histologie der sogenannten ,akuten Leukämie". Virchows Arch. f. pathol. Anat. u. Physiol. u. f. klin. Med. I906, Bd. I84, S. 220. 


\section{Erklärung der Abbildungen auf Tafel I.}

Fig. r. Präparat aus Milz. Hämatoxylin-Eosinfärbung. Zeiss Okular I, Objektiv AA. Haufen kleiner Lymphocyten inmitten der großen Lymphocyten.

Fig. 2. Präparat aus Leber. Hämatoxylin-Eosinfärbung. Zeiss Okular I, Objektiv AA. Interazinös, in der Umgebung der Gefäße befindliche große Lymphocyten.

Fig. 3. Präparat aus Lymphom am Halse. Hämatoxylin-Eosinfärbung. Zeiss Okular I, Objektiv AA. Alle Schichten einer Gefäßwand in einem lymphoiden Herde von den großen Lymphocyten durchsetzt. 
Deutsche Zeitschrift f. Chirurgie 132.Bd.

Tafel I.

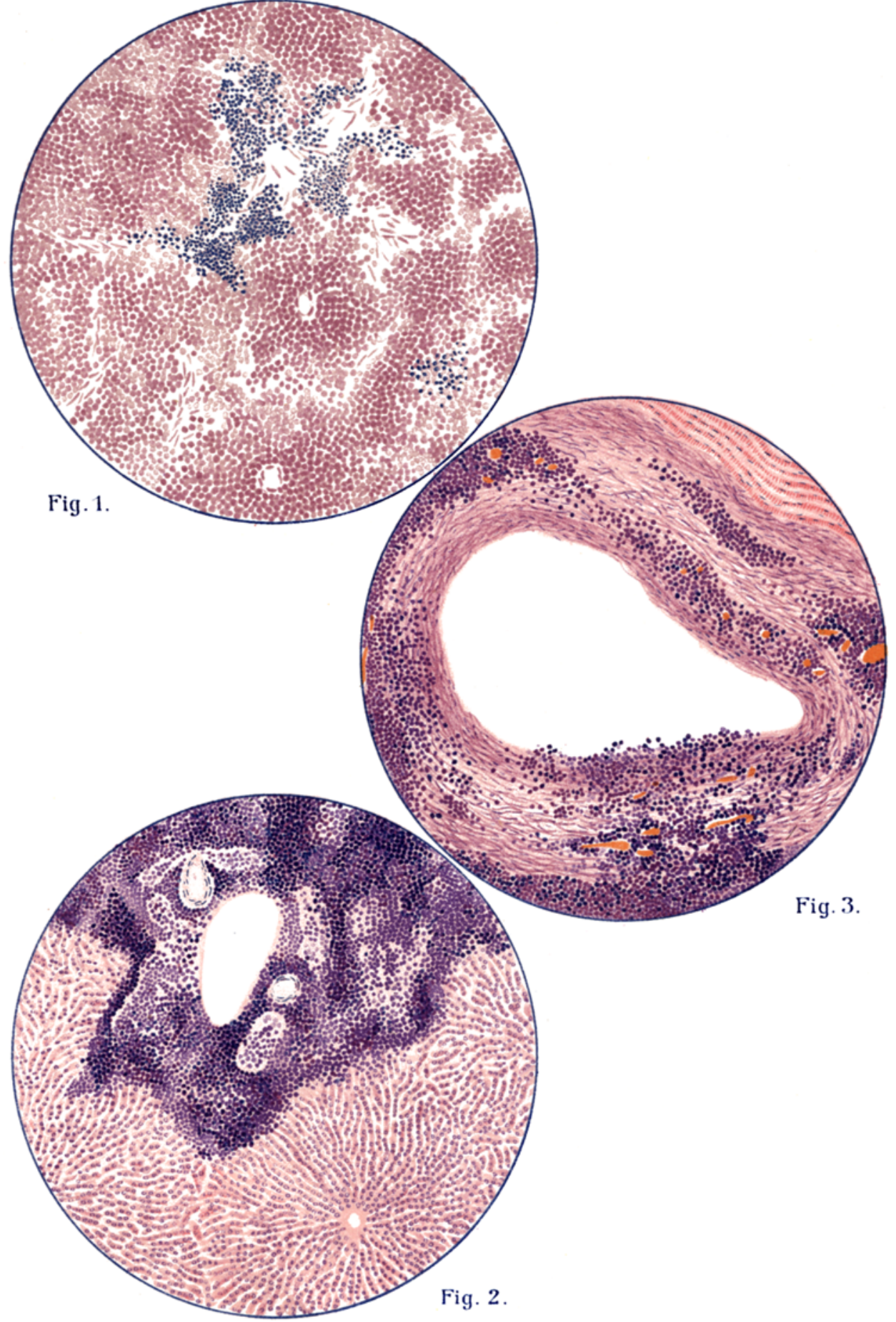

